\title{
80-dB Microwave Noise from an Avalanche Transistor Circuit
}

\author{
Gytis Mykolaitis ${ }^{1}$, Saulius Kersulis ${ }^{2}$, Skaidra Bumeliene ${ }^{2}$, Arunas Tamasevicius ${ }^{2}$ \\ ${ }^{I}$ Department of Physics, Vilnius Gediminas Technical University, \\ Sauletekio Al. 11, LT-10223 Vilnius, Lithuania \\ ${ }^{2}$ Institute of Semiconductor Physics, Center for Physical Sciences and Technology, \\ A. Gostauto St. 11, LT-01108 Vilnius, Lithuania \\ gytis.mykolaitis@vgtu.lt
}

\begin{abstract}
Extremely high 'excess noise ratio' of $80 \mathrm{~dB}$ is observed from an avalanche transistor circuit, operating in a random pulse mode. Broadband noise spectrum measured from $30 \mathrm{MHz}$ to $1 \mathrm{GHz}$ exhibits good flatness with the nonuniformity of only $\pm 1 \mathrm{~dB}$. Experiments have been performed with the silicon bipolar junction microwave transistors. An analog circuit model is proposed and investigated.
\end{abstract}

Index Terms-Bipolar transistor circuits, microwave transistors, avalanche breakdown, noise generators.

\section{INTRODUCTION}

Electronic circuits, employing bipolar junction transistors biased into the avalanche breakdown region are known for many years [1], [2]. A variety of circuit modifications with a number of applications to switching devices, nanosecond and subnanosecond pulse generators have been developed. Commonly the pulse generators, when supplied with voltage $V_{0}$ noticeably above the threshold voltage $V_{t h}$ of the collector-base junction $\left(V_{0}>V_{t h}\right)$, produce periodic pulses [3]-[7]. In this paper, we describe an avalanche transistor circuit operating in a random pulse mode, which is observed at supply voltages very close to the threshold, $V_{0} \approx V_{t h}$.

\section{EXPERIMENTAL RESULTS}

The circuit diagram is sketched in Fig. 1. The transistor Q is a silicon bipolar microwave BFG520 type $\left(f_{T}=9 \mathrm{GHz}, V_{t h}\right.$ $\approx 26 \mathrm{~V}$ ) device. The values of the passive elements are the following: $C_{1}=1 \mathrm{pF}, R_{1}=24 \mathrm{k} \Omega, R_{2}=1 \mathrm{k} \Omega, R_{3}=50 \Omega$.

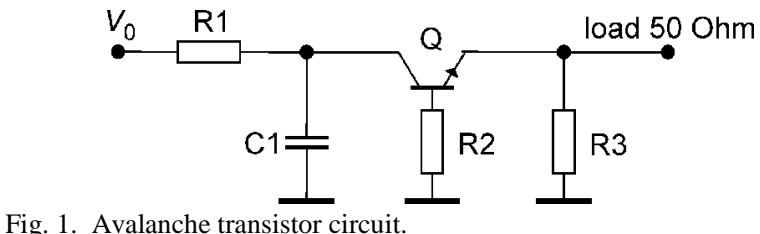

Pulse trains, recorded by means of a digital oscilloscope Tektronix DPO71604C (bandwidth $16 \mathrm{GHz}$ ), are presented in Fig. 2 and Fig. 3, for random and periodic mode of operation, respectively (note different time scales in Fig. 2 and Fig. 3). An individual pulse is shown in Fig. 4. Its amplitude and shape do not depend on the applied voltage; they are the same in the random and the periodic modes.
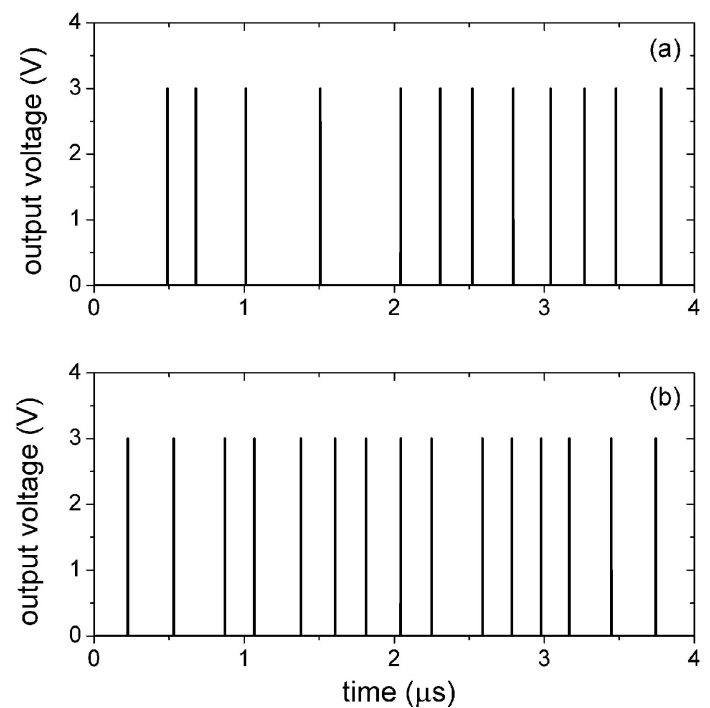

Fig. 2. Two snapshots (a) and (b) of random pulses. $V_{0}=26 \mathrm{~V}$.

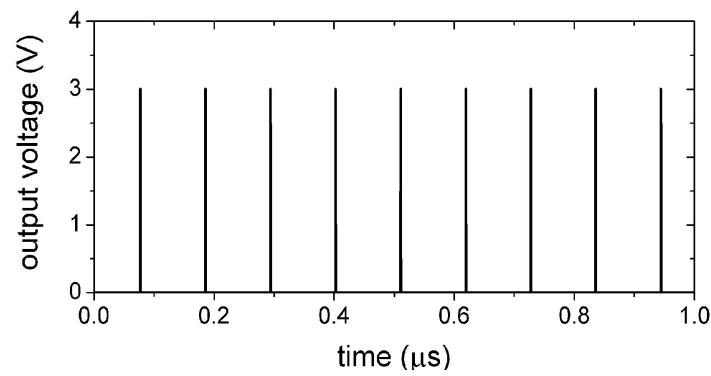

Fig. 3. Periodic pulse train. $V_{0}=28.5 \mathrm{~V}$.

For the pulse trains examined above, corresponding noise level commonly can be characterized by the power spectral density $k_{B} T_{n}$ (in $\mathrm{W} / \mathrm{Hz}$ ), where $k_{B}$ is the Boltzmann constant, $T_{n}$ is the effective noise temperature (in $\mathrm{K}$ ). The output level in the specifications of noise devices is conventionally characterized by the 'excess noise ratio' $(E N R)$ either in a linear or in a logarithmic scale, respectively

$$
E N R=\frac{T_{n}-T_{0}}{T_{0}},
$$




$$
E N R=10 \log _{10} \frac{T_{n}-T_{0}}{T_{0}}, \quad[\mathrm{~dB}]
$$

where $T_{0}$ is the standard temperature $(290 \mathrm{~K})$.

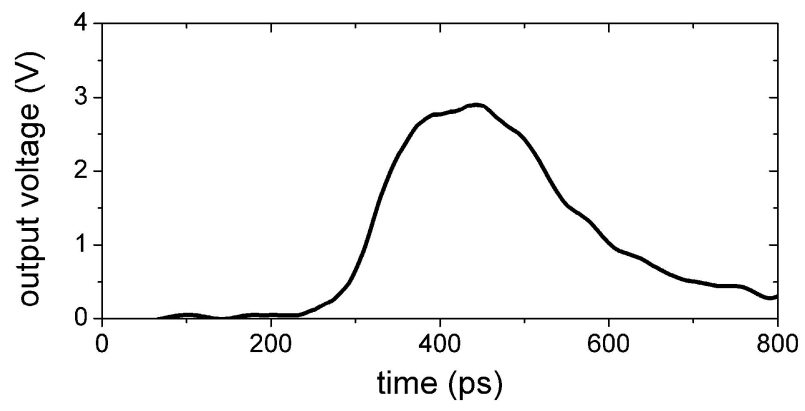

Fig. 4. Experimentally recorded individual pulse.

The spectral distribution of the $\operatorname{ENR}(\omega)$ can be estimated from the shape of an individual pulse (Fig. 4), which we approximate by the Gaussian function

$$
V(t)=V_{m} \exp \left[-\frac{1}{2}\left(\frac{t-t_{0}}{\tau}\right)^{2}\right],
$$

where $V_{m}=3 \mathrm{~V}, t_{0}=430 \mathrm{ps}$, and $\tau=100 \mathrm{ps}$. Then the envelope of the ENR spectrum is given by

$$
\operatorname{ENR}(\omega)=\operatorname{ENR}(0) \exp \left((\omega \tau)^{2} / 2\right)
$$

where $\omega=2 \pi f$. Based on the above analytical expression, the spectral density at $f=1 \mathrm{GHz}$ decreases to the level of $0.8 \times$ $\operatorname{ENR}(0)$.

The experimental power spectrum is presented in Fig. 5.

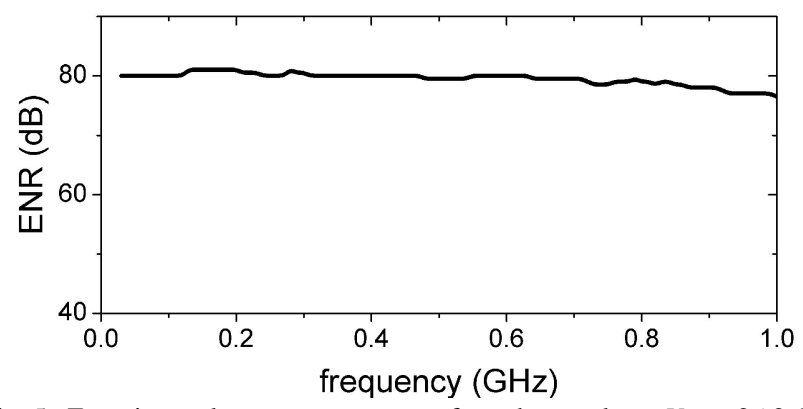

Fig. 5. Experimental power spectrum of random pulses. $V_{0}=26.2 \mathrm{~V}$. Spectral resolution $120 \mathrm{kHz}$.

TABLE I. NOISE PARAMETERS FOR DIFFERENT SOURCES.

\begin{tabular}{|l|c|c|c|}
\hline Noise source & Ref. & $\boldsymbol{T}_{\boldsymbol{n}}(\mathbf{K})$ & $\boldsymbol{E N R}(\mathbf{d B})$ \\
\hline Gas discharge tube & {$[8]$} & $2.5 \times 10^{3}-3 \times 10^{4}$ & $8.8-20.1$ \\
\hline Avalanche diode & {$[9]$} & $1.5 \times 10^{5}-1 \times 10^{6}$ & $26-35$ \\
\hline $\begin{array}{l}\text { Microplasma } \\
\text { diode }\end{array}$ & {$[10]$} & $1 \times 10^{8}$ & 55 \\
\hline $\begin{array}{l}\text { Avalanche } \\
\text { transistor }\end{array}$ & Fig. 5 & $3 \times 10^{10}$ & 80 \\
\hline Chaotic oscillator & {$[11],[12]$} & $3 \times 10^{11}$ & 90 \\
\hline
\end{tabular}

The power spectrum (Fig. 5) demonstrates extremely high, $80 \mathrm{~dB}$ spectral density and rather flat spectral distribution $( \pm 1 \mathrm{~dB})$. The decrease to the level of $0.5 \times$
$E N R(0)$, i.e. by $-3 \mathrm{~dB}$ at $1 \mathrm{GHz}$ is in a reasonable agreement with the estimation from (3). Noise level $\left(T_{n}\right.$ and $\left.E N R\right)$ for the avalanche transistor circuit is compared with some other noise sources in Table I.

The microplasma diode in Table I refers to GaAs avalanche diode, biased below the breakdown voltage and periodically driven by external microwave voltage at $1 \mathrm{GHz}$ [10]. The chaotic oscillator [11], [12] is the two-stage Colpitts circuit with the same microwave silicon BFG520 type transistors, operating in the $300 \mathrm{MHz}-1000 \mathrm{MHz}$ range.

\section{ANALOG MODEL}

To demonstrate the mechanism behind the observed random pulses in the avalanche transistor circuit, we propose a phenomenological analog model, which is presented in Fig. 6 as an electronic circuit. The R1-C1 subcircuit plays the same role of charge accumulating unit as in the experimental circuit (Fig. 1). The operational amplifier OA stage is a common buffer used to minimize leakage from the capacitor $\mathrm{C}$ 1. The comparator unit COMP with a positive feedback via the resistors R2 and R3 implements a hysteretic Schmitt trigger [13]. The ON and the OFF threshold input voltages of the Schmitt trigger are given by:

$$
\begin{aligned}
& V_{\text {on }}=V_{h} \frac{R_{2}}{R_{2}+R_{3}}+\left(E+V_{\text {noise }}\right) \frac{R_{3}}{R_{2}+R_{3}}, \\
& V_{\text {off }}=V_{l} \frac{R_{2}}{R_{2}+R_{3}}+\left(E+V_{\text {noise }}\right) \frac{R_{3}}{R_{2}+R_{3}} .
\end{aligned}
$$

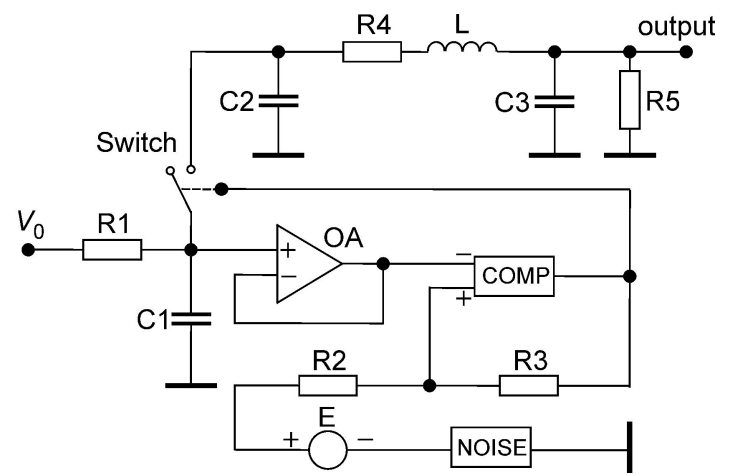

Fig. 6. Analog low frequency circuit, modelling the microwave avalanche transistor circuit in Fig. 1.

In (5), (6) the $V_{h}$ and the $V_{l}$ are the high level and the low level output voltage of the comparator, respectively (specifically, $V_{h}=13 \mathrm{~V}, V_{l}=0.2 \mathrm{~V}$ ), the $E$ is the dc bias voltage from the voltage source $\mathrm{E}$, the $V_{\text {noise }}$ is the voltage from the external NOISE unit. The $V_{\text {noise }}$ is the frequency dependent signal, filtered from a broadband random noise source by means of a low-pass first-order RC filter at the cut-off frequency of $500 \mathrm{~Hz}$. The root mean square noise voltage is about $10 \mathrm{mV}$. At $f>500 \mathrm{~Hz}$ the noise level decreases by a factor of 2 per octave. Thus, it can be considered as $1 / \mathrm{f}$ noise. For $R_{2}=R_{3}=5.6 \mathrm{k} \Omega$ and $E=10 \mathrm{~V}$, used in the model circuit, the $V_{\text {on }}=11.5 \mathrm{~V}$, the $V_{\text {off }}=5.1 \mathrm{~V}$ (assuming $\left|V_{\text {noise }}\right|<<E$ ).

The Schmitt trigger and the electronically controlled switch implement a unit with an $S$-type current-voltage 
characteristic, typically observed in a transistor operating in an avalanche mode [1]-[6]. The subcircuit C2-R4-L-C3R5 is inserted in the model circuit to imitate the inertial properties of the avalanche transistor. The values of the elements of the circuit in Fig. 6 are: $C_{1}=22 \mathrm{nF}, R_{1}=24 \mathrm{k} \Omega$, $L=5 \mathrm{mH}, C_{2}=C_{3}=10 \mathrm{nF}, R_{4}=R_{5}=510 \Omega$.

All variable voltages from the model circuit have been recorded by means of a digital oscilloscope Tektronix TDS2014B (bandwidth $100 \mathrm{MHz}$ ). The power spectra have been measured using a low frequency analog spectrum analyzer with the spectral resolution of $10 \mathrm{~Hz}$. The voltage across capacitor $\mathrm{C} 1$ saturates to $V_{0}$, which is set slightly less than the ON threshold input voltage of the Schmitt trigger, $V_{0} \leq V_{o n}$. The trigger waits for a noise signal of negative sign to lower, according to (5), the $V_{\text {on }}$ so that $V_{0} \geq V_{\text {on }}$. Then the output of the trigger changes from high to low level and turns on the electronic switch. The latter discharges capacitor $\mathrm{C} 1$ and produces the Gaussian shaped output pulse (Fig. 7) with $\tau \approx 10 \mu \mathrm{s}$.

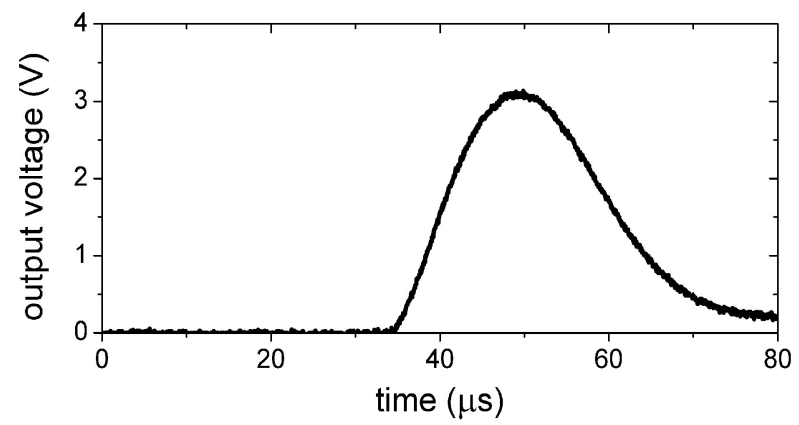

Fig. 7. Individual pulse from the model circuit in Fig. 6.

At higher voltages, $V_{0}>V_{\text {on }}$ the influence of noise is unimportant and periodic pulses are observed (Fig. 8).

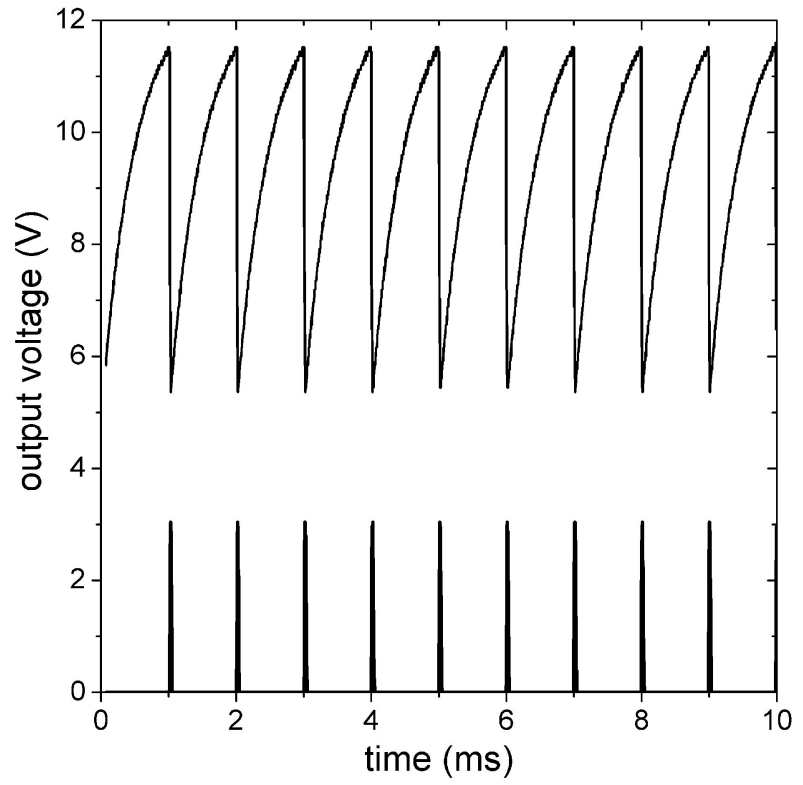

Fig. 8. Periodic pulses from the model circuit; voltage across capacitor $\mathrm{C} 1$, taken from the output of the buffer OA (top trace), and output voltage (bottom trace). $V_{0}=12.5 \mathrm{~V}$.

The random pulse trains, shown in Fig. 9(a) and Fig. 9(b), are observed at supply voltages $V_{0}$ very close to the threshold voltage, $V_{0} \approx V_{\text {on }}=11.5 \mathrm{~V}$. Since noise is a random process, the time intervals between the output pulses are also random, providing broadband continuous spectrum
(Fig. 10). The time parameter in (3) of an individual pulse $\tau=10 \mu \mathrm{s}$. Formula (4) gives an estimate, that $\operatorname{ENR}(f)$ decreases to the level of 0.8 at $f=10 \mathrm{kHz}$.
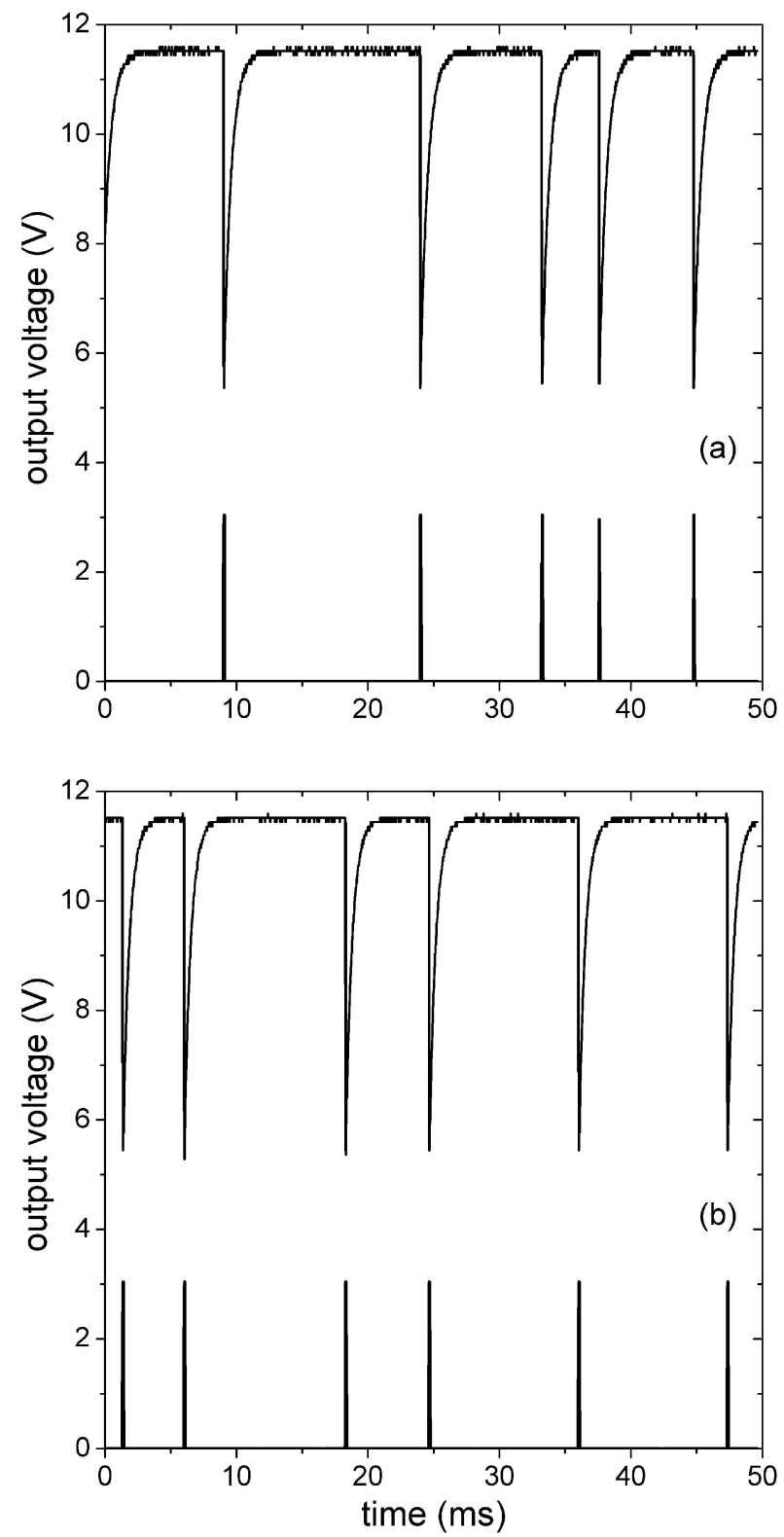

Fig. 9. Two snapshots (a) and (b) of random pulses from the model circuit; voltage across capacitor $\mathrm{C} 1$, taken from the output of the buffer OA (top traces) and output voltage (bottom traces). $V_{0}=11.5 \mathrm{~V}$.

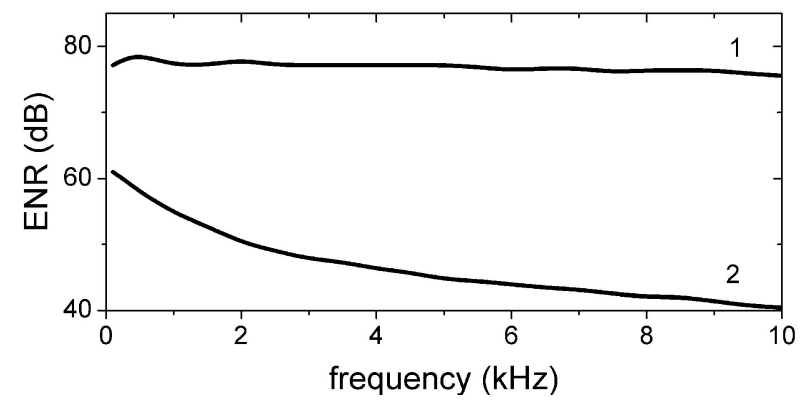

Fig. 10. Power spectra from the model circuit; spectrum of the output signal (1), and spectrum of the noise unit (2). $V_{0}=11.5 \mathrm{~V}$.

\section{CONCLUSIONS}

We have demonstrated, that broadband high spectral density noise with "excess noise ratio" value up to $80 \mathrm{~dB}$, covering the very high (VHF: $30 \mathrm{MHz}-300 \mathrm{MHz}$ ) and the 
ultrahigh (UHF: $300 \mathrm{MHz}-1000 \mathrm{MHz}$ ) frequency bands, can be generated by an avalanche transistor circuit operating in a random pulse mode. The excess noise ratio is much higher than of many other noise sources and is only by $10 \mathrm{~dB}$ lower than of the chaotic waveform oscillators, e.g. the two-stage Colpitts circuit [11], [12]. Whereas, the avalanche transistor random pulse circuit has a big advantage over the chaotic microwave oscillators in respect of the spectrum flatness. Chaotic oscillators generate very high power, up to $90 \mathrm{~dB}$, but have a very serious shortcoming, because of the nonuniformity of the spectra, typically of about $20 \mathrm{~dB}$ to $30 \mathrm{~dB}$.

Some recently described chaotic oscillators demonstrate rather uniform spectra [14], [15]. However, their versions, developed so far, operate at low (kilohertz) frequencies only. The avalanche transistor circuit, presented in this paper, exhibits high noise level in the microwave range and good flatness of the spectrum with the nonuniformity of $\pm 1 \mathrm{~dB}$.

An important observation, made by Dyakonov et al. [6], is that not only the transistors, specially designed as the avalanche devices, but many bipolar junction transistors exhibit good performance in the avalanche breakdown region. The silicon bipolar microwave BFG520 type transistor, investigated in this paper, is another example.

To explain the experimental results we have suggested and investigated an analog model, specifically a low frequency electrical circuit, which generates random signals very similar to the pulses, observed experimentally from the avalanche transistor circuit. The time scale in the model circuit, compared to the experimental one, has been changed by a factor of $10^{5}$ : the time parameter $\tau$ is increased from $100 \mathrm{ps}$ to $10 \mu \mathrm{s}$. Consequently, the corresponding cut-off frequency, where the spectrum drops to the level of 0.8 , is decreased from $1 \mathrm{GHz}$ to $10 \mathrm{kHz}$. However, the shape of the individual pulse (Fig. 7) and the shape of the power spectrum (Fig. 10, curve 1), obtained from the analog model, agree very well with the experimental plots, shown in Fig. 4 and Fig. 5, respectively.

Similar approach using analog modelling method has been introduced by Namayunas et al. [16] and has exhibited very good agreement with the experimental observations. The analog modelling technique (in a sense an analog computer) can be applied to microplasma noise phenomenon [17] to explain the effect of noise enhancing by means of breakdown stimulation with microwave electric field in avalanche diodes.

\section{REFERENCES}

[1] D. J. Hamilton, J. F. Gibbons, W. Shockley, "Physical principles of avalanche transistor pulse circuits", in Proc. IRE, vol. 47, no. 6, 1959, pp. 1102-1108. [Online]. Available: http://dx.doi.org/10.1109/ isscc.1959.1157029

[2] W. M. Henebry, "Avalanche transistor circuits", Rev. Sci. Instr., vol. 32, no. 11, pp. 1198-1203, 1961.

[3] P. Spirito, G. F. Vitale, "An analysis of the dynamic behavior of switching circuits using avalanche transistors", IEEE J. Solid-State Circuit, vol. SC-7, no. 4, pp. 315-320, 1972.

[4] V. P. Dyakonov, "Nanosecond rectangular pulse generators using avalanche and MIS transistors", Instrum. Exp. Tech., vol. 23, no. 4, pp. 913-914, 1980.

[5] V. P. Dyakonov, "Nanosecond pulse generators based on avalanche and MIS transistors", Instrum. Exp. Tech., vol. 24, no. 1, pp. 131-132, 1981.

[6] V. P. Dyakonov, T. A. Vasilkova, Yu. A. Ermachkova, "Measurement of the pulse parameters of silicon transistors under avalanche operating conditions", Meas. Tech., vol. 50, no. 7, pp. 770-774, 2007.

[7] G. Duan, S. Vainshtein, J. Kostamovaara, "Three-dimensional peculiarities in an avalanche transistor provide a broadened range of amplitudes and durations in the generated pulses", Appl. Phys. Lett., vol. 101, no. 17, pp. 173-506, 2012. [Online]. Available: http://dx.doi.org/10.1063/1.4764114

[8] TD/TN series microwave noise tubes and noise sources. [Online] Available: http://www.highenergydevices.com

[9] Calibrated sources: NC3000 coaxial. [Online] Available: http://noisecom.com

[10] A. Namajunas, A. Tamasevicius, G. Mykolaitis, S. Bumeliene, J. Pozela, "Microplasma noise stimulated by microwave electric field", Acta Phys. Polon. A, vol. 107, no. 2, pp. 369-372, 2005.

[11] A. Tamasevicius, G. Mykolaitis, S. Bumeliene, A. Cenys, A. N. Anagnostopolous, E. Lindberg, "Two-stage chaotic Colpitts oscillator", Electron. Lett., vol. 37, no. 9, pp. 549-551, 2001.

[12] S. Bumeliene, A. Tamasevicius, G. Mykolaitis, A. Baziliauskas, E. Lindberg, "Numerical investigation and experimental demonstration of chaos from two-stage chaotic Colpitts oscillator in the ultrahigh frequency range", Nonlinear Dyn., vol. 44, no. 1-4, pp. 167-172, 2006.

[13] P. Horowitz, W. Hill, The Art of Electronics. Cambridge, New York: Cambridge University Press, 1993, pp. 231-232.

[14] A. Tamasevicius, S. Bumeliene, R. Kirvaitis, G. Mykolaitis, E. Tamaseviciute, E. Lindberg, "Autonomous Duffing-Holmes type chaotic oscillator", Elektronika ir Elektrotechnika, no. 5, pp. 43-46, 2009.

[15] E. Tamaseviciute, G. Mykolaitis, A. Tamasevicius, "Autonomous Silva-Young chaotic oscillator with flat power spectrum", Elektronika ir Elektrotechnika, no. 9, pp. 109-112, 2011.

[16] A. M. Namayunas, Yu. K. Pozhela, A. V. Tamashyavichyus, "Microplasma instability in alternating electric field", Sov. Phys. Dokl., vol. 34, pp. 630-632, 1989.

[17] J. Pozhela, Plasma and Current Instabilities in Semiconductors, Oxford, New York: Pergamon, 1981. 